\title{
MicroRNA-17-5p: At the Crossroads of Cancer and Aging - A Mini-Review
}

\author{
Hanna Dellago Madhusudhan Reddy Bobbili Johannes Grillari \\ Christian Doppler Laboratory on Biotechnology of Skin Aging, Department of Biotechnology, BOKU - University of \\ Natural Resources and Life Sciences Vienna, Vienna, Austria
}

\section{Key Words}

miRNA $\cdot$ miR-17-5p · Aging $\cdot$ Longevity $\cdot$ Biomarker .

Cancer

\begin{abstract}
The miR-17-92 cluster, led by its most prominent member, miR-17-5p, has been identified as the first miRNA with oncogenic potential. Thus, the whole cluster containing miR-17$5 p$ has been termed oncomiR-1. It is strongly expressed in embryonic stem cells and has essential roles in vital processes like cell cycle regulation, proliferation and apoptosis. The importance of miR-17-5p for fundamental biological processes is underscored by the fact that a miR17-deficient mouse is neonatally lethal. Recently, miR-17-5p was identified in the context of aging, since it is comprised in a common signature of miRNAs that is downregulated in several models of aging research. Recently, miR-17-5p turned out to be the first 'longevimi $R$ ' in an animal model, extending the lifespan of a transgenic miR-17-5p-overexpressing mouse. Here, we summarize the current status of research on miR$17-5 p$ with emphasis on its role in cellular senescence, aging and cancer, which points to a pleiotropic function of miR-17$5 p$ regulating multiple targets involved in autophagy, cell cycle regulation and apoptosis in a tissue-dependent fashion. In addition, its elevated presence in serum or plasma of a wide range of tumor patients suggests using it as an 'alarm-
\end{abstract}

\section{KARGER}

E-Mail karger@karger.com www.karger.com/ger
(C) 2016 The Author(s)

Published by S. Karger AG, Basel

Karger
Open access

This article is licensed under the Creative Commons AttributionNonCommercial-NoDerivatives 4.0 International License (CC BYNC-ND) (http://www.karger.com/Services/OpenAccessLicense) Usage and distribution for commercial purposes as well as any distribution of modified material requires written permission.
iR', a general indicator of a potential tumor pathology. However, amounts of circulating miR-17-5p of healthy individuals as reference values are still missing, before any miRNA can be classified as such an 'alarmiR'. In conclusion, miR-17$5 p$ is at the crossroads of aging, longevity and cancer and might represent a promising biomarker or even therapeutic tool and target in this context.

(c) 2016 The Author(s)

Published by S. Karger AG, Basel

\section{Introduction}

The increasing mean lifespan of the population is a big success story of humanity, but also poses a challenge that industrialized countries are currently facing, since aging is associated with increased susceptibility to many diseases like cancer, type 2 diabetes, neurodegenerative disorders and steatohepatitis. Aging is considered to be caused by limitations in somatic maintenance as a tradeoff to reproduction, resulting in an accumulation of molecular and consequently tissue and organ damage over time [1]. Multiple mechanisms that cause or promote damage to macromolecules, cells and tissues, kept at bay by an equal number of mechanisms counteracting, pre-

H.D. and M.R.B. contributed equally to this work.
Johannes Grillari

Christian Doppler Laboratory on Biotechnology of Skin Aging, Department of Biotechnology, BOKU - University of Natural Resources and Life Sciences Vienna Muthgasse 18, AT-1190 Vienna (Austria)

E-Mail Johannes.grillari@boku.ac.at 
venting and repairing damage, have to be elucidated to develop a complete understanding of the aging process.

One prominent mechanism strongly related with cellular aging is cellular senescence. Senescence represents a permanent growth arrest induced by telomere attrition, oncogenic or environmental stress that functions a tumor-suppressor mechanism. Besides, senescent cells are now increasingly appreciated for their role in embryonic development, wound healing and tissue repair [2]. However, as senescent cells accumulate during lifetime, they exert detrimental effects on tissue function, contribute to chronic inflammatory states, and thus contribute to tissue, organ and organismal aging [3], and removal of naturally occurring senescent cells in the mouse postpones the development of age-related pathologies and extends the lifespan [4].

However, precise molecular understanding is still scarce, especially concerning miRNAs, which have been established as an important layer of gene expression control and might represent an attractive target for interventions aiming at healthy aging. Biogenesis, function and mechanisms of action of miRNAs are reviewed in great detail (e.g. [5]). In brief, one miRNA is able to regulate up to hundred mRNA targets and therefore potentially orchestrates a large variety of cellular processes similar to transcription factors [6,7], contributing even to a potential posttranscriptional operon concept [8].

The miR-17-92 cluster comprises some of the beststudied miRNAs so far. This cluster contains 6 miRNA members with overlapping and specific roles. Here, we focus on miRNA-17-5p and its role in aging, age-related diseases and cancer, emphasizing the emerging role of 'longevimiR'-17 in cellular and organismal aging.

\section{Transcriptional Regulation and Target mRNAs}

\section{Conservation and Regulation of the miRNA-17-92 Cluster}

The miR-17-92 cluster is located in the locus of the nonprotein-coding gene MIR17HG on chromosome 13. The miR-17-92 transcript spans 800 nucleotides within its host gene and comprises six miRNAs - miR-17-5p, miR-18a, miR-19a, miR-20a, miR-19b-1 and miR-92a-1. Its sequence is highly conserved among vertebrates [9], especially in the seed region (fig. 1a), as also shown by phylogenetic tree analysis (fig. 1b). No homologues are found in nonvertebrates so far according to miRbase [10].

In addition, paralogous versions of $\mathrm{miR}-17$ have arisen by intragenomic gene duplication in the miR-106a-363 and miR-106b-25 clusters. While the seed regions are well conserved (fig. 1c), differences in the $3^{\prime}$ and $5^{\prime}$ regions suggest overlapping, but different sets of target mRNAs and thus functions. How is the miR-17-92 cluster regulated on a transcriptional level? So far, several regulators of miR-17-92 transcription have been described.

$\mathrm{c}-\mathrm{Myc}$, the cellular homolog of the retroviral v-myc oncogene, is a well-studied proto-oncogene. Besides regulating transcription of a plethora of protein-coding genes, c-myc activates the miR-17-92 cluster and represses a dozen of other miRNAs. Interestingly, a number of c-myc transcriptional targets (fig. 2), like RPS6KA5 (ribosomal protein S6 kinase, $90 \mathrm{kDa}$, polypeptide 5), BCL11B (B-cell CLL/lymphoma 11B), PTEN (phosphatase and tensin homolog), E2F1 (E2F transcription factor 1) and HCFC2 (host cell factor $\mathrm{C} 2$ ) are also predicted targets of the miR-17-92 cluster [11], which suggests a delicate regulatory mechanism by which c-myc simultaneously activates transcription and restricts abundance of respective mRNAs.

A similar mechanism is thought to be at work between Aurora Kinase A (AURKA), the miR-17-92 cluster and the transcription factor E2F1. AURKA is a serine/threonine kinase essential for regulation of mitosis and is overexpressed in many cancers. AURKA stabilizes the transcription factor E2F1 by inhibiting its proteasomal degradation (fig. 2), which again induces miR-17-92 transcription [12] and is at the same time its target.

p53 acts as negative transcriptional regulator of the miR-17-92 cluster. Under hypoxia, p53 represses miR17-92 transcription and thereby sensitizes cells for hypoxia-induced apoptosis, while overexpression of the miR-17-92 cluster inhibits apoptosis under hypoxia [13].

Results of the ENCODE (Encyclopedia of DNA Elements) project further revealed that along with these experimentally confirmed transcription factors, BCL3 (Bcell CLL/lymphoma 3), IRF1 (interferon regulatory factor 1), SP1 (Sp1 transcription factor), TAL1 (T-cell acute lymphocytic leukemia 1) and ZBTB33 (zinc finger and BTB domain containing 33) might regulate the miR-1792 cluster (fig. 2) and are also targeted by individual miRNAs of the cluster [14].

On the posttranscriptional level, several studies find that the miR-17-92 cluster members are not expressed to equal degrees in cells and tissues as would be expected from their joint transcription as a primary miRNA. This might be due to factors that differentially protect or degrade the single miRNA members after the pre-miRNA status, as it has been published for miR-18 [15]. However, so far, no such reports exist on miR-17-5p. In terms of 


\title{
Multiple sequence alignment of miR-17-5p in different species
}

\author{
Shsa-miR-17-5p M10000071 \\ GUCAGAAUAAUGUCAAAGUGCUUACAGUGCAGGUAGUGAUAUGUGCAUCUACUGCAGUGAAGGCACUUGUAGCAUUAUGGUGAC \\ miR-17-5p \\ miR-17-3p
}
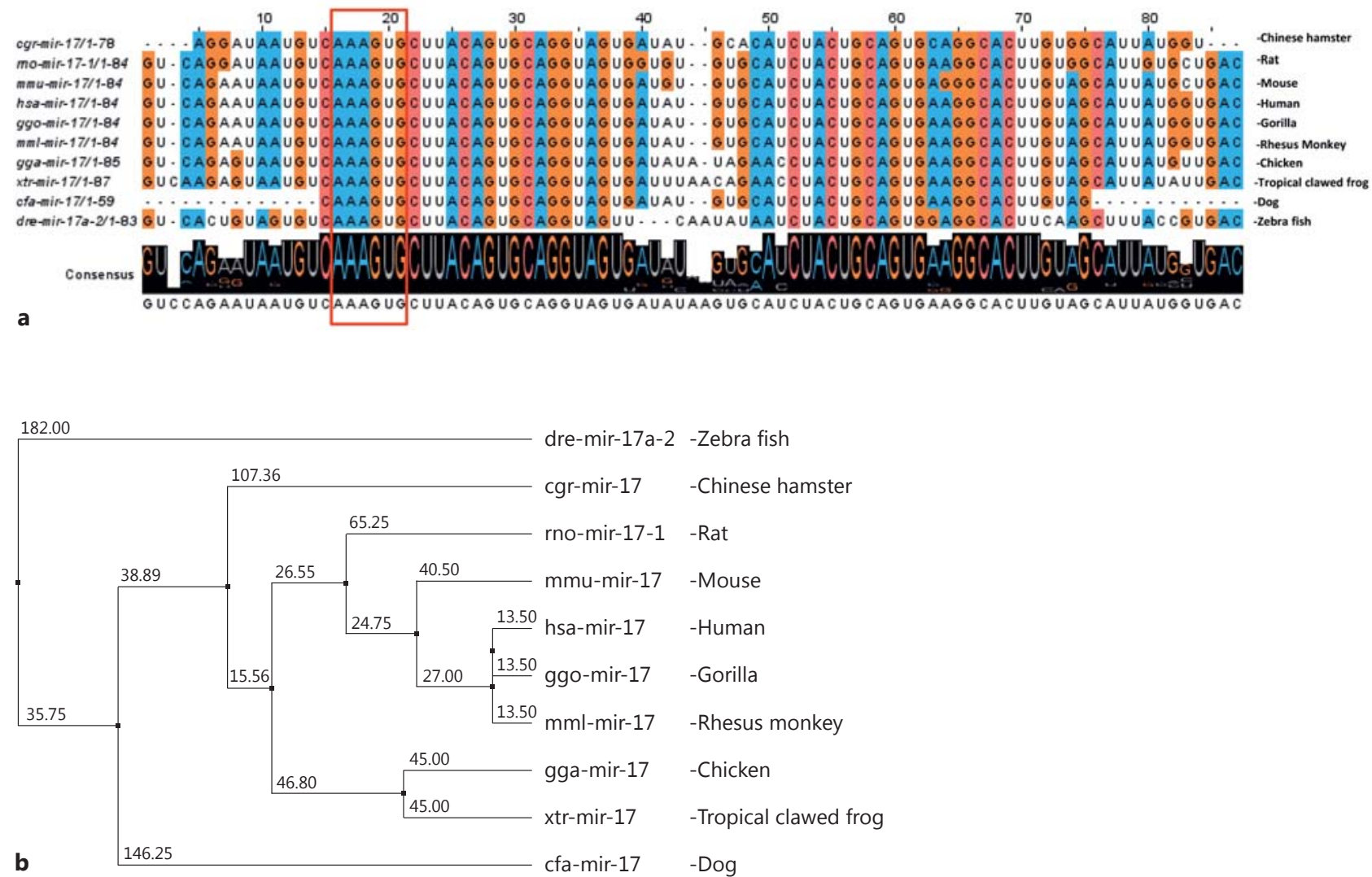

30

50

60

70

80

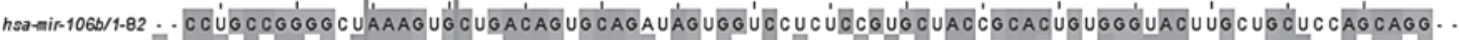
$h s a-m i m-17 / 1-84$ GUCAGAAUAAUGUCAAAGUGCUUACAGUGCAGGUAGUGAUAUG - UGCAUCUACUGCAGUGAAGOCACUUGUAGCAUUAUGGUGAC hsa-mir-106a/1-81 - CCUUGOC CAUGUAAAAGUG CUUACAQUGCAGQUAGCUUUUUG - A. GAUCUACUGCAAUGUAAGCACUUCUUACAUUACCAUGG .

Consensus

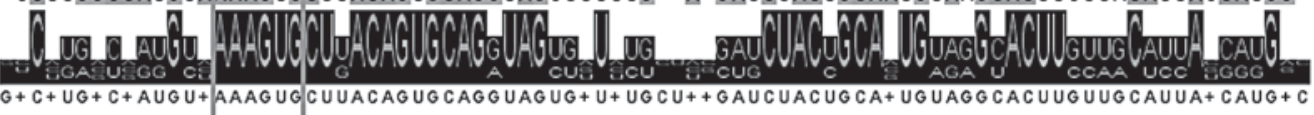

C

Fig. 1. Sequence similarities of miR-17-5p. a Phylogenetic comparison of miR-17-5p shows almost $100 \%$ sequence identity in monkey and gorilla and a high degree of similarity down to Zebra fish. $\mathbf{b}$ miR-17-5p is conserved among vertebrates. The numbers in the phylogenetic tree indicate the evolutionary distance between the organisms. c miR-17-5p has two homologous miRNAs encoded in the miR-106a-363 and miR-106b-25 clusters. The consensus sequence of these 3 seed family members, i.e. mature sequences with identical seed region, is shown. 


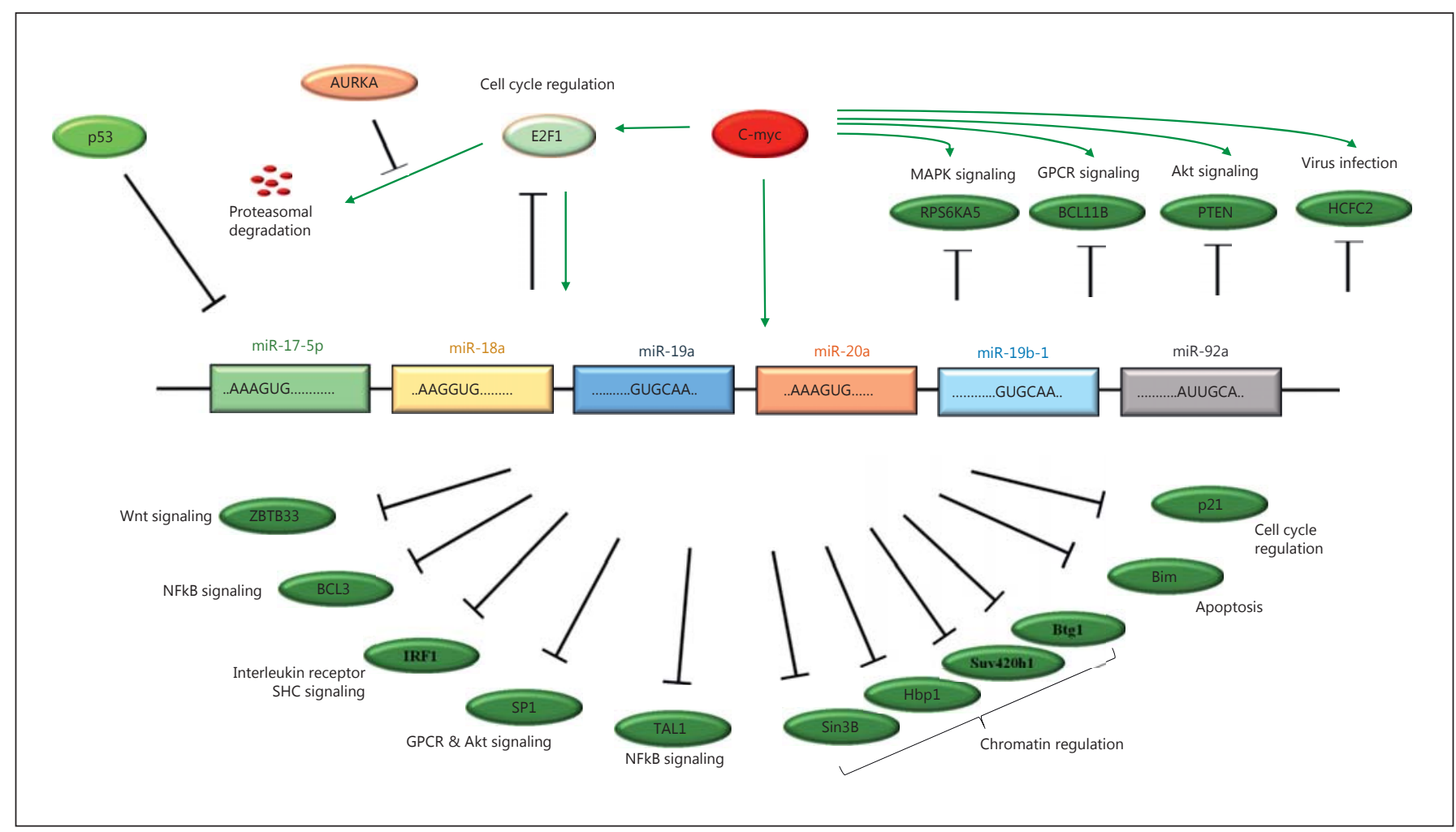

Fig. 2. The miR-17-92 regulatory network. Expression of the miR17-92 cluster is controlled by multiple upstream regulators, some of which are in turn directly or indirectly targeted by miR-17-92 cluster members. miR-17-92 expression is induced by c-myc, and several c-myc transcriptional targets like RPS6KA5, BCL11B, PTEN, E2F1 and HCFC2 are targeted by miR-17-92, guaranteeing a delicate balance of miRNA and protein abundance. AURKA = Aurora kinase $\mathrm{A}$; Akt $=\mathrm{V}$-Akt murine thymoma viral oncogene homolog; BCL3 = B-cell CLL/lymphoma 3; BCL11B = B-cell CLL/ lymphoma 11B; Bim = BCL2 like 11; Btg1 = B-cell translocation gene 1, antiproliferative; E2F1 = E2F transcription factor 1; Hbp1 = HMG-box transcription factor 1; GPCR = G protein-cou-

tissue and cell type specificity, miR-17-5p is expressed ubiquitously and highly in all tissues tested so far [16], pointing to a generally high importance of this miRNA.

\section{Target mRNAs of the miR-17-92 Cluster and miR-17-5p}

PTEN and E2Fs were the first confirmed targets of the miR-17-92 cluster in the context of cell cycle progression and apoptosis [17]. By now, a multitude of targets of the miR-17-92 cluster have been reported including members of the TGF $\beta$ (transforming growth factor- $\beta$ ) signaling pathway [18]. In addition, it also targets specific chromatin regulatory genes, such as Sin $3 \mathrm{~b}$ (SIN3 transcription regulator family member $\mathrm{B}$; a transcriptional repressor pled receptor; $\mathrm{HCFC} 2$ = host cell factor $\mathrm{C} 2$; IRF1 = interferon regulatory factor 1 ; MAPK = mitogen-activated protein kinase; myc $=\mathrm{V}-\mathrm{Myc}$ avian myelocytomatosis viral oncogene homolog; $\mathrm{NFkB}=$ nuclear factor kappa $\mathrm{B} ; \mathrm{p} 21$ = cyclin-dependent kinase inhibitor 1A; p53 = tumor protein P53; PTEN = phosphatase and tensin homolog; RPS6KA5 = ribosomal protein S6 kinase, $90 \mathrm{kDa}$, polypeptide 5; SHC = Src homology 2 domain containing transforming protein 1 ; Sin $3 \mathrm{~b}=$ SIN3 transcription regulator family member B; SP1 = Sp1 transcription factor; Suv420h1 = suppressor of variegation 4-20 homolog 1; TAL1 $=$ T-cell acute lymphocytic leukemia 1; Wnt = wingless-type MMTV integration site family; ZBTB33 = zinc finger and BTB domain containing 33 .

for MYC-responsive genes), Hbp1 (HMG-box transcription factor 1; a transcriptional repressor that negatively regulates the Wnt-mediated beta catenin signaling pathway), Suv420h1 (suppressor of variegation 4-20 homolog 1; a histone methyltransferase, targeted to histone $\mathrm{H} 3$ by retinoblastoma proteins), and Btg1 (B-cell translocation gene 1, antiproliferative; a regulator of cell growth and differentiation), as well as the apoptosis regulator Bim (Bcl-2 interacting mediator of cell death; an activator of neuronal and lymphocyte apoptosis) [19] and the cell cycle inhibitor p21 (fig. 2) [20]. Hence, miR-17-92 seems to maintain cell proliferation and survival, which might result in a neoplastic or progenitor/regenerative state of cells [19]. 
A series of mouse strains with targeted deletions of individual miR-17-92 members and subsequent phenotypic and gene expression analysis have recently provided a detailed picture of how miR-17-92 affects gene expression in vivo [21].

In the following, we recapitulate data that point to a dual role of this cluster that might well result in an antagonistic pleiotropic function of miR-17-5p in young versus elderly individuals.

\section{Aging, the miR-17-92 Cluster, and miR-17-5p}

\section{Aging-and Senescence-Dependent Regulation of \\ miR-17-5p}

In recent years, the important role of miRNAs in aging became increasingly evident [22]. In an effort to identify miRNAs commonly regulated during aging, microarray studies were performed comparing four human replicative cell-aging models - endothelial cells, skin fibroblasts, T-cells and renal proximal tubular epithelial cells - as well as three organismal aging models comprising skin, Tcells and mesenchymal stem cells. These very different model systems shared a set of commonly downregulated miRNAs, among them members of the miR-17-92 cluster including miR-17-5p, miR-19b, miR-20a and miR-106a [23]. Several studies using different model systems have confirmed the downregulation of the miR-17-92 cluster during aging [24], and its upregulation in centenarians who are considered successful agers $[25,26]$.

These expression changes are recapitulated by stressinduced senescence, as microarray data revealed that members of the miR-17-92 cluster, including miR-17-5p, are downregulated in stress-induced senescence of human diploid fibroblasts and human trabecular meshwork cells [27]. Figure 3a gives an overview over up- and downregulation of miR-17-5p expression during aging and age-related diseases.

\section{A Long-Lived Pre-miR-17-Overexpressing Mouse Model}

The interest in the role of miR-17-5p in aging research has peaked with the report that its overexpression in mouse extends the organismal lifespan by approximately $16 \%$ [28]. This finding was somewhat surprising after the same authors had previously observed that miR-17 represses fibronectin expression, leading to cellular defects, growth retardation, smaller organs and strongly reduced hematopoietic cell lineages in miR-17-overexpressing mice [29], results that do not seem well reconciled yet.
However, in favor of its life-prolonging role, miR-17$5 p$ targets IRS1 (Insulin Receptor Substrate 1) and ADCY 5 (Adenylate Cyclase 5), which modulate a complex signaling network, leading to upregulation of genes involved in autophagy and repressing senescence and apoptosis (fig. 3b) [28].

To be more precise, IRS1 on the one hand activates AKT, which suppresses the mTOR inhibitor AMPK. Therefore, miR-17-5p-dependent silencing of IRS1 abrogates AMPK suppression, enabling mTOR inhibition and thus activating autophagy. On the other hand, IRS1 promotes expression of FOXO3A (forkhead box O3) and LC3- $\beta$ (microtubule-associated protein 1 light chain 3 beta), which also enhance autophagy. At the same time, repression of miR-17-5p's direct target ADCY5 leads to translocation of RGS2 (regulator of G-protein signaling 2) from the membrane into the nucleus, where it interacts with and activates transcription of MKP7 (mitogen-activated protein kinase phosphatase 7). The MKP7 protein dephosphorylates PRAS40 (40-kDa proline-rich AKT substrate) and mTOR (mechanistic target of rapamycin), which thereupon bind each other, resulting in a suppression of mTOR activity that in consequence leads to attenuation of protein synthesis and stimulation of autophagy, resulting in reduced senescence. In fact, miR-17 transgenic mice exhibited increased bone mass and decreased presence of senescent cells in the skin, intestine, lung and heart. In addition, MKP7 acts as a negative regulator of c-Jun amino-terminal kinase and extracellular signal-regulated kinase (ERK) pathways. ERKs function in the control of cell division, and inhibitors of these enzymes are potential anticancer agents, hence miR-17-5p might also extend the organismal life span by reducing cancer risk, but that is still subject to speculation, since - as stated above miR-17 transgenic mice develop liver tumors [28]. The authors do not give any information about the causes of death in their miR-17 mice, and a potential confounder might be that both miR-17-5p and miR-17-3p are expressed from the pre-miR-17 construct to a comparable degree, and hence the relative effect of each specific miRNA to the observed organismal phenotype cannot be determined [28].

Only few other miRNAs have been described to postpone cellular or organismal aging, one of them being inhibition of miR-21 in endothelial cell senescence [30]. Since elongation of the life span is still considered the golden standard for proving an activity in the aging process, we suggest tagging such miRNAs with the label 'longevi-miR'.
Dellago/Bobbili/Grillari 
Fig. 3. miR-17-5p in aging and age-related diseases. a Overview of miR-17-5p expression levels during aging and age-related pathologies. $\uparrow$ and $\downarrow$ designate up- and downregulation, respectively. b Relationship between miR-17-5p and mTOR, a central regulator of autophagy and apoptosis. miR-17-5p targets ACDY5, causing translocation of the GTPase activator RGS2 from the membrane to the nucleus, where it induces expression of MKP7. MKP7 dephosphorylates mTOR, which thereupon dimerizes with PRAS40 and is inactivated. Inhibitory phosphorylation of the ULK1ATG13-FIP200 heterotrimer by mTOR is consequently abolished and activating phosphorylation catalyzed by AMPK. Consequently, the ULK1 complex activates autophagy, which in turn attenuates senescence and apoptosis. Simultaneously, miR-17-5p targets IRS1. IRS1 can activate AKT via PI3K, and AKT inhibits the autophagy activator AMPK. Therefore, IRS1 downregulation by miR-17-5p activates autophagy via two pathways. AKT $=$ Proto-oncogene c-Akt; AMPK = AMP-activated protein kinase; ATG13 = autophagy related 13; FIP200 = focal adhesion kinase family kinase-interacting protein of $200 \mathrm{kDa}$; IRS1 = insulin receptor substrate 1; MKP7 = mitogen-activated protein kinase phosphatase 7; PRAS40 = 40-kDa proline-rich AKT substrate; RISC = RNA-induced silencing complex; ULK1 = Unc-51 like autophagy activating kinase 1.

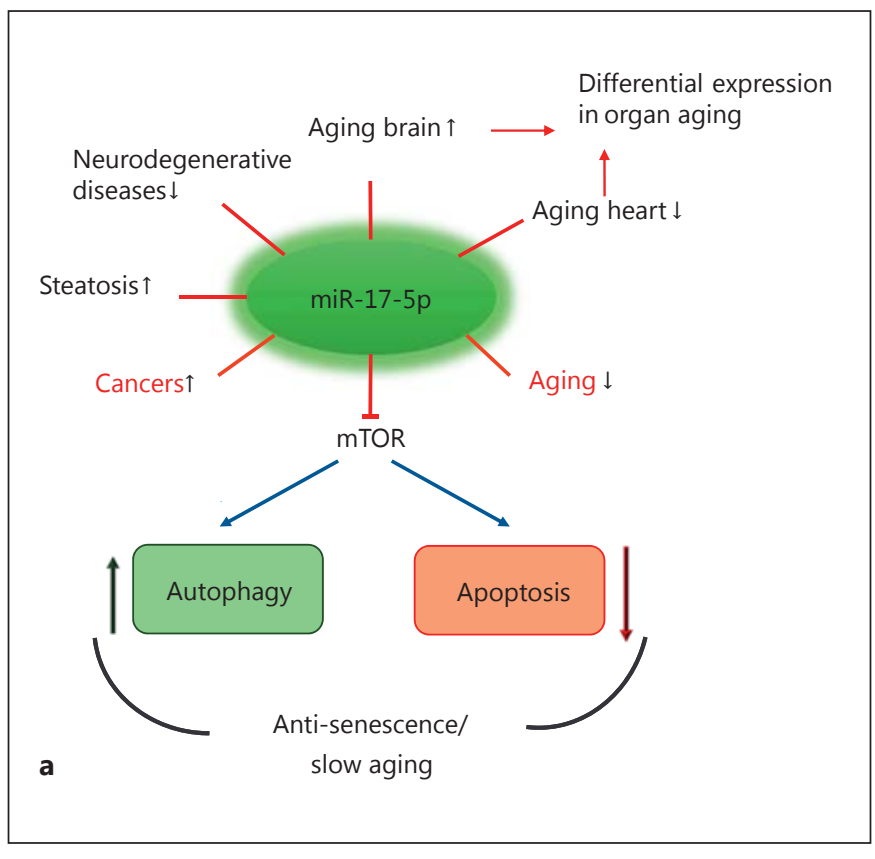

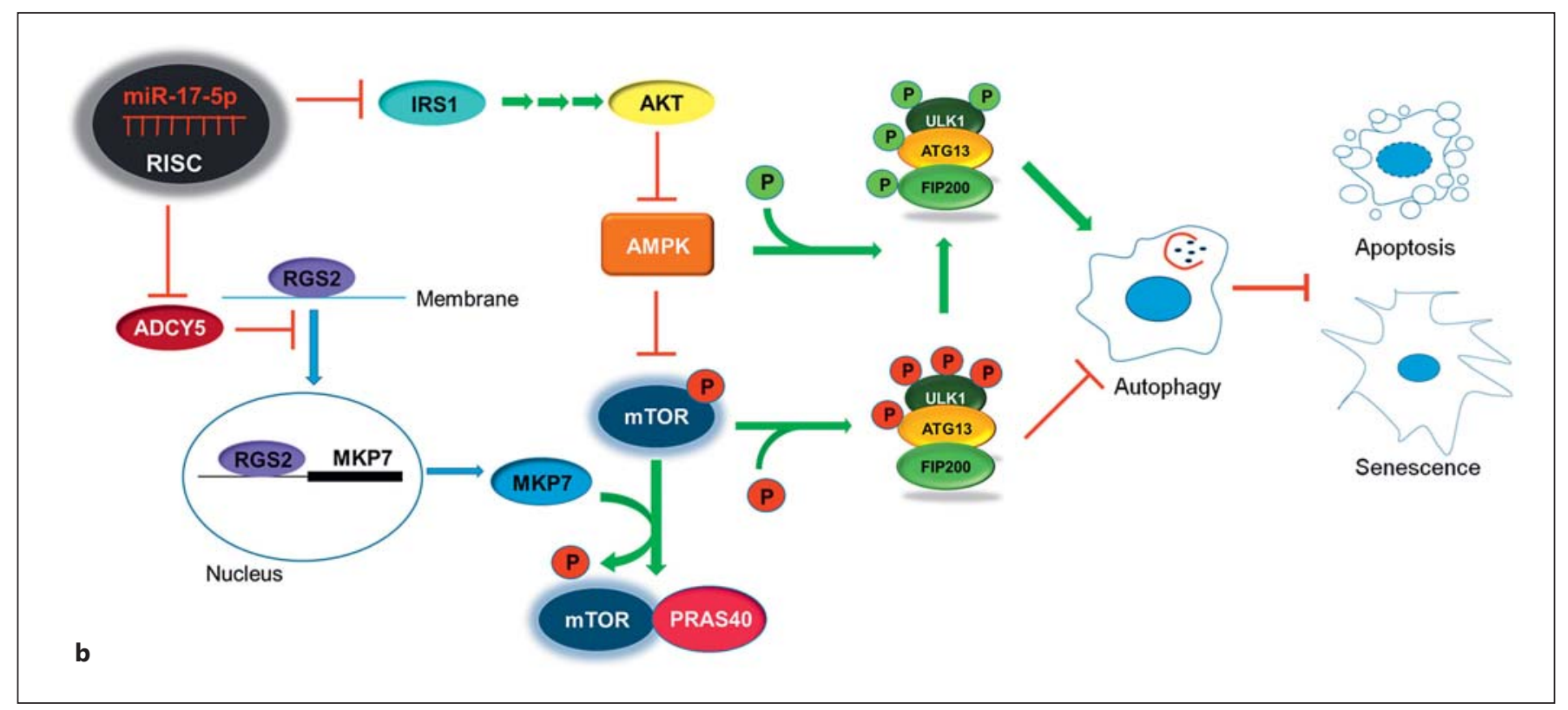

\section{Cardiac Function and miR-17-5p}

In transgenic mice, miR-17-5p is upregulated in damaged heart regions after induced infarction. It directly targets two inhibitors of matrix metalloproteases (MMPs), TIMP1 (tissue inhibitor of metalloproteinases 1) and TIMP2 (tissue inhibitor of metalloproteinases 2), leading to matrix remodeling after infarction [31]. In turn, miR-17 inhibition in a mouse model in vivo decreased MMP activity, enhanced cardiac function and helped prevent heart failure after infarction. Similarly, miR-17-5p is protective in a kidney ischemia-reperfusion model [32].

In addition, overexpression of pre-miR-17 in mouse cardiac fibroblasts enhances cell survival upon oxidative stress, increases proliferation and reduces senescenceassociated $\beta$-galactosidase staining [33], whereby the miR-17-3p component of the pre-miR-17 activates a transcriptional program that promotes epithelial-to- 
mesenchymal transition and self-renewal while suppressing senescence, and might even act as a miR-17-5p antagomiR.

In summary, miR-17-5p and $-3 p$ play a role in cardiac aging in transgenic mice and cells in vitro. Again, both miR-17-5p and miR-17-3p are expressed from the same pre-miR-17, and therefore the relative contribution of each miRNA to the observed phenotype is not clear.

\section{Aging, the Bone and miR-17-5p}

The miR-17-92 cluster plays an important role in bone formation, as knockout in mice results in reduced bone mineral content and reduced bone strength [34]. Furthermore, miR-17-5p overexpression promotes osteogenic differentiation of MSCs (mesenchymal stem cells) [35]. However, during aging and in pro-inflammatory diseases, miR-17-5p expression was observed low in MSCs, concomitant with compromised osteogenic differentiation [36]. miR-17 overexpression partially rescued this defect by repressing Smurf1 (Smad ubiquitin regulatory factor one) [37] and SMAD7.

Since low miR-17-92 expression is a hallmark of aging and senescence [23], and senescent cells contribute to reduced osteogenesis $[38,39]$, it might be speculated that it is involved in loss of bone mineral density in the elderly. However, data on senescence and bone disease are still scarce, while one hint into this direction is that telomerase-deficient mice lose bone mass, most probably due to the SASP (senescence-associated secretory phenotype) [40].

\section{miR-17-5p in the Aging Brain}

Surprisingly, miR-17 expression is increased in the brains of old mice compared to young mice [41], while the relative abundance of the majority of miRNAs tested decreased in the mouse brain during aging. The informative value of this study is limited, though, by the fact that data are based on pooled cDNA libraries derived from only 2 individuals for each condition. Such an unexpected increase might be interpreted in the context of astrogliosis. Astrogliosis (or reactive astrocytosis) is an abnormal increase in the number of astrocytes due to the destruction of nearby neurons. It can be observed in age-related neurodegenerative diseases like Alzheimer's disease [42] or after spinal cord injury (SCI), where it leads to glial scar formation and inhibits axonal regeneration. miR-17-5p is upregulated after SCI and contributes to astrocyte proliferation [43] and hence potentially also to the astrogliosis phenotype observed in age-related neurodegenerative diseases.
miR-17-5p and mTOR - A Connection between

Proliferation, Cancer and Aging

One focus in research on interventions that slow aging is on the mammalian target of rapamycin (mTOR). Inhibition of mTOR is one of the few accepted and universal interventions in model organisms that extends the lifespan and postpones age-related diseases like cardiovascular diseases, cancer and diabetes, and mTOR inhibitors are widely used to treat these diseases. Inhibition of mTOR activates many pathways that upregulate autophagy. Thereby, it fulfills multiple purposes - the clearance of dysfunctional or unnecessary molecules or organelles, the provision of nutrients and the degradation of intracellular pathogens. Both normal and premature aging are accompanied by a decline in autophagic potential, and a lack of functional autophagy leads to tissue degeneration similar to that observed in aged tissue [44]. In addition, mTOR is a central coordinator of cell proliferation whose function is deregulated in many cancers. Therefore, inhibitors of mTOR like rapamycin and its analogues are potential antitumor agents, some already approved for clinical use in cancer therapy.

Given its importance in cell physiology and tissue maintenance, it comes as no surprise that the mTOR pathway is subject to regulation by miRNAs, among them also the miR-17-92 cluster [45].

As shown in figure $3 \mathrm{~b}$, inhibition of $\mathrm{mTOR}$ by miR17-5p upregulates autophagy and slows down the aging process in the mouse model presented by Du et al. [28], discussed in the section A Long-Lived Pre-miR-17 Overexpressing Mouse Model. Indeed, oncogenic miRNAs like miR-17 are upregulated in rapamycin-resistant cells and inhibition of miR-17 restored rapamycin sensitivity. Thus, inhibitors of miR-17 could potentially serve as adjuvants in chemotherapy. However, it becomes clear from these studies that miR-17-5p is at the crossroads between aging and cancer.

\section{miR-17-5p and Its Role in Cancer}

In general, miR-17-5p is considered as an oncogene, supported by a large body of evidence from many different tumors. However, it might also be 'guilty-by-association' with its oncogenic miR-17-92 cluster members [46], especially miR-19, which has been postulated as having the highest oncogenic potential [47]. By now, a more differentiated view has emerged in literature, where miR-17$5 \mathrm{p}$ alone possesses metastasis suppressor functions [48]. In addition, it seems to suppress cancer growth by stimu- 
lating T cells [49]. Finally, high circulating levels of miR17-5p have been found in serum of patients suffering of several different types of cancer [50,51], suggesting that high levels of circulating miR-17-5p might serve as an alarm signal, or 'alarmiR'.

In sum, the effect of miR-17-5p varies according to cancer type, model system, and probably also to the relative expression levels of miR-17-3p and miR-17-5p, depending on the constructs used for overexpression and knockdown. The tumor-suppressive and tumorigenic properties of the miR-17-92 cluster are summarized in more detail in other recent reviews, e.g. $[52,53]$.

\section{Conclusion}

In this review, we focused on the role of miR-17-5p in cellular senescence, aging and age-related diseases and opposed this role to cancer. miR-17-5p plays a major role in regulating many genes involved in autophagy, apoptosis and cell cycle regulation. Its overexpression in the mouse model extends the lifespan by promoting autophagy, and hence we suggest terming miR-17-5p the first 'longevi-miRNA'. At the same time, circulating miR-17$5 p$ levels turned out to be high in almost all cancers, atherosclerosis and obesity, and might act as a biomarker or alarming signal, meriting the designation 'alarmiR-17'. While miR-17-5p is ubiquitously expressed, its biological function seems very cell, tissue and disease specific, which accounts for the confusingly high number of reports suggesting it to have a dual role, as a tumor suppressor and as an oncogene. Not only miR-17-5p, but also miR-17-3p surprisingly plays a role as tumor suppressor and as oncogene, sometimes synergistically with miR-17-5p, sometimes independently. Therefore, more research is needed to understand the basic biology of miR-17-3p and miR$17-5 p$ in order to establish them as prognostic, diagnostic and therapeutic targets for aging and age-related diseases as well as cancer.

\section{Acknowledgements}

This work was funded by the BioToP - 'Biomolecular technology of proteins' PhD Programme, Austrian Science Funds (FWF) Project W1224, and the Christian Doppler Society. The financial support by the Austrian Federal Ministry of Economy, Family and Youth, the National Foundation for Research, Technology and Development as well as the FP7 EU projects Frailomic and Sybil are also gratefully acknowledged.

We apologize to all the authors who contributed to today's state of knowledge in the field and to whom we could not give credit due to the limited numbers of references.

\section{Disclosure Statement}

J.G. is a co-founder of Evercyte $\mathrm{GmbH}$ and TAmiRNA GmbH.

\section{References}

1 Kirkwood TB: Understanding the odd science of aging. Cell 2005; 120:437-447.

$\checkmark 2$ Demaria M, Ohtani N, Youssef SA, Rodier F, Toussaint W, Mitchell JR, et al: An essential role for senescent cells in optimal wound healing through secretion of PDGF-AA. Dev Cell 2014;31:722-733.

-3 Coppé J-P, Desprez P-Y, Krtolica A, Campisi $\mathrm{J}$ : The senescence-associated secretory phenotype: the dark side of tumor suppression. Annu Rev Pathol 2010;5:99-118.

-4 Baker DJ, Childs BG, Durik M, Wijers ME, Sieben CJ, Zhong J, et al: Naturally occurring p16Ink4a-positive cells shorten healthy lifespan. Nature 2016;530:184-189.

-5 Jonas S, Izaurralde E: Towards a molecular understanding of microRNA-mediated gene silencing. Nat Rev Genet 2015;16:421-433.

6 Stefani G, Slack FJ: Small non-coding RNAs in animal development. Nat Rev Mol Cell Biol 2008;9:219-230.
7 Weilner S, Schraml E, Redl H, Grillari-Voglauer R, Grillari J: Secretion of microvesicular miRNAs in cellular and organismal aging. Exp Gerontol 2013;48:626-633.

-8 Fischer S, Mathias S, Schaz S, Emmerling VV, Buck T, Kleemann M, et al: Enhanced protein production by microRNA-30 family in $\mathrm{CHO}$ cells is mediated by the modulation of the ubiquitin pathway. J Biotechnol 2015;212:3243.

9 Mendell JT: miRiad roles for the miR-17-92 cluster in development and disease. Cell 2008; 133:217-222.

10 Kozomara A, Griffiths-Jones S: miRBase: annotating high confidence microRNAs using deep sequencing data. Nucleic Acids Res 2014;42:D68-D73.

11 Lewis BP, Shih I, Jones-Rhoades MW, Bartel DP, Burge CB: Prediction of mammalian microRNA targets. Cell 2003;115:787-798.
12 He S, Yang S, Deng G, Liu M, Zhu H, Zhang $\mathrm{W}$, et al: Aurora kinase A induces miR-17-92 cluster through regulation of E2F1 transcription factor. Cell Mol Life Sci 2010;67:20692076.

13 Yan H, Xue G, Mei Q, Wang Y, Ding F, Liu M-F, et al: Repression of the miR-17-92 cluster by p53 has an important function in hypoxia-induced apoptosis. EMBO J 2009;28: 2719-2732.

14 Gerstein MB, Kundaje A, Hariharan M, Landt SG, Yan K-K, Cheng C, et al: Architecture of the human regulatory network derived from ENCODE data. Nature 2012;489:91-100.

15 Guil S, Cáceres JF: The multifunctional RNAbinding protein hnRNP A1 is required for processing of miR-18a. Nat Struct Mol Biol 2007;14:591-596.

-16 Kaya KD, Karakülah G, Yakicier CM, Acar AC, Konu O: mESAdb: microRNA expression and sequence analysis database. Nucleic Acids Res 2011;39:D170-D180. 
17 Cloonan N, Brown MK, Steptoe AL, Wani S, Chan WL, Forrest ARR, et al: The miR-17-5p microRNA is a key regulator of the G1/S phase cell cycle transition. Genome Biol 2008; 9:R127.

18 Petrocca F, Vecchione A, Croce CM: Emerging role of miR-106b-25/miR-17-92 clusters in the control of transforming growth factor beta signaling. Cancer Res 2008;68:81918194.

19 Li Y, Choi PS, Casey SC, Dill DL, Felsher DW: MYC through miR-17-92 suppresses specific target genes to maintain survival, autonomous proliferation, and a neoplastic state. Cancer Cell 2014;26:262-272.

20 Fontana L, Fiori ME, Albini S, Cifaldi L, Giovinazzi S, Forloni M, et al: Antagomir17-5p abolishes the growth of therapy-resistant neuroblastoma through p21 and BIM. PLoS One 2008;3:e2236.

-21 Han Y, Vidigal JA, Mu P, Yao E, Singh I, González AJ, et al: An allelic series of miR17 92 - mutant mice uncovers functional specialization and cooperation among members of a microRNA polycistron. Nat Genet 2015;47:766-775.

-22 Grillari J, Grillari-Voglauer R: Novel modulators of senescence, aging, and longevity: Small non-coding RNAs enter the stage. Exp Gerontol 2010;45:302-311.

-23 Hackl M, Brunner S, Fortschegger K, Schreiner C, Micutkova L, Mück C, et al: miR-17, miR-19b, miR-20a, and miR-106a are downregulated in human aging. Aging Cell 2010;9: 291-296.

-24 Dhahbi JM, Atamna H, Boffelli D, Magis W, Spindler SR, Martin DIK: Deep sequencing reveals novel MicroRNAs and regulation of MicroRNA expression during cell senescence. PLoS One 2011;6:e20509.

-25 Serna E, Gambini J, Borras C, Abdelaziz KM, Mohammed K, Belenguer A, et al: Centenarians, but not octogenarians, up-regulate the expression of microRNAs. Sci Rep 2012;2: 961.

-26 Gombar S, Jung HJ, Dong F, Calder B, Atzmon G, Barzilai N, et al: Comprehensive microRNA profiling in B-cells of human centenarians by massively parallel sequencing. BMC Genomics 2012;13:353.

-27 Li G, Luna C, Qiu J, Epstein DL, Gonzalez P: Alterations in microRNA expression in stress-induced cellular senescence. Mech Ageing Dev 2009;130:731-741.

28 Du WW, Yang W, Fang L, Xuan J, Li H, Khorshidi A, et al: miR-17 extends mouse lifespan by inhibiting senescence signaling mediated by MKP7. Cell Death Dis 2014;5:e1355.
29 Shan SW, Lee DY, Deng Z, Shatseva T, Jeyapalan Z, Du WW, et al: MicroRNA MiR-17 retards tissue growth and represses fibronectin expression. Nat Cell Biol 2009;11:10311038 .

30 Dellago H, Preschitz-Kammerhofer B, Terlecki-Zaniewicz L, Schreiner C, Fortschegger $\mathrm{K}$, Chang MW, et al: High levels of oncomiR-21 contribute to the senescence induced growth arrest in normal human cells and its knock-down increases the replicative life span. Aging Cell 2013;12:446-458.

31 Li S-H, Guo J, Wu J, Sun Z, Han M, Shan SW, et al: miR-17 targets tissue inhibitor of metalloproteinase 1 and 2 to modulate cardiac matrix remodeling. FASEB J 2013;27:4254-4265.

- 32 Kaucsár T1, Révész C, Godó M, Krenács T, Albert M, Szalay CI, Rosivall L, Benyó Z, Bátkai S, Thum T, Szénási G, Hamar P: Activation of the miR-17 family and miR-21 during murine kidney ischemia-reperfusion injury. Nucleic Acid Ther 2013;23:344-354.

33 Du WW, Li X, Li T, Li H, Khorshidi A, Liu F, et al: The microRNA miR-17-3p inhibits mouse cardiac fibroblast senescence by targeting Par4. J Cell Sci 2015;128:293-304.

34 Mohan S, Wergedal JE, Das S, Kesavan C: Conditional disruption of miR17-92 cluster in collagen type I-producing osteoblasts results in reduced periosteal bone formation and bone anabolic response to exercise. Physiol Genomics 2015;47:33-43.

35 Jia J, Feng X, Xu W, Yang S, Zhang Q, Liu X, et al: MiR-17-5p modulates osteoblastic differentiation and cell proliferation by targeting SMAD7 in non-traumatic osteonecrosis 2014;46:e107-e108.

36 Liu W, Qi M, Konermann A, Zhang L, Jin F, Jin Y: The p53/miR-17/Smurf1 pathway mediates skeletal deformities in an age-related model via inhibiting the function of mesenchymal stem cells. Aging (Albany NY) 2015; $7: 205-216$.

37 Zhu H, Kavsak P, Abdollah S, Wrana JL, Thomsen GH: A SMAD ubiquitin ligase targets the BMP pathway and affects embryonic pattern formation. Nature 1999;400:687-693.

38 Weilner S, Grillari-Voglauer R, Redl H, Grillari J, Nau T: The role of microRNAs in cellular senescence and age-related conditions of cartilage and bone. Acta Orthop 2015;86:9299.

39 Weilner S, Schraml E, Wieser M, Messner P, Schneider K, Wassermann K, et al: Secreted microvesicular miR-31 inhibits osteogenic differentiation of mesenchymal stem cells. Aging Cell 2016, Epub ahead of print.
40 Saeed H, Abdallah BM, Ditzel N, Catalalehnen P, Qiu W, Amling M, et al: Telomerase-deficient mice exhibit bone loss owing to defects in osteoblasts and increased osteoclastogenesis by inflammatory microenvironment. J Bone Miner Res 2011;26:1494-1505.

41 Inukai S, de Lencastre A, Turner M, Slack F: Novel microRNAs differentially expressed during aging in the mouse brain. PLoS One 2012; 7:e40028.

42 Rodríguez-Arellano JJ, Parpura V, Zorec R, Verkhratsky A: Astrocytes in physiological aging and Alzheimer's disease. Neuroscience 2016;323:170-182.

43 Hong P, Jiang M, Li H: Functional requirement of dicer 1 and miR-17-5p in reactive astrocyte proliferation after spinal cord injury in the mouse. Glia 2014;62:2044-2060.

44 Rubinsztein DC, Mariño G, Kroemer G: Autophagy and aging. Cell 2011;146:682-695.

45 Totary-Jain H, Sanoudou D, Ben-Dov IZ, Dautriche CN, Guarnieri P, Marx SO, et al: Reprogramming of the microRNA transcriptome mediates resistance to rapamycin. J Biol Chem 2013;288:6034-6044.

46 Hayashita Y, Osada H, Tatematsu Y, Yamada $\mathrm{H}$, Yanagisawa $\mathrm{K}$, Tomida S, et al: A polycistronic microRNA cluster, miR-17-92, is overexpressed in human lung cancers and enhances cell proliferation. Cancer Res 2005;65: 9628-9632.

-47 van Haaften G, Agami R: Tumorigenicity of the miR-17-92 cluster distilled. Genes Dev 2010;24:1-4

48 Fan M, Sethuraman A, Brown M, Sun W, Pfeffer LM: Systematic analysis of metastasis-associated genes identifies miR-17-5p as a metastatic suppressor of basal-like breast cancer. Breast Cancer Res Treat 2014;146:487-502.

49 Li H, Gupta S, Du WW, Yang BB: MicroRNA-17 inhibits tumor growth by stimulating T-cell mediated host immune response. Oncoscience 2014;1:531-539.

50 Wang $\mathrm{M}, \mathrm{Gu} \mathrm{H}$, Wang S, Qian H, Zhu W, Zhang L, et al: Circulating miR-17-5p and miR-20a: molecular markers for gastric cancer. Mol Med Rep 2012;5:1514-1520.

51 Zeng X, Xiang J, Wu M, Xiong W, Tang H, Deng M, et al: Circulating miR-17, miR-20a, miR-29c, and miR-223 combined as non-invasive biomarkers in nasopharyngeal carcinoma. PLoS One 2012;7:e46367.

52 Fuziwara CS, Kimura ET: Insights into regulation of the miR-17-92 cluster of miRNAs in Cancer. Front Med 2015;2:64.

53 Mendell JT: miRiad roles for the miR-17-92 cluster in development and disease. Cell 2008; 133:217-222. 